\title{
OBSERVATIONS OF X-RAY JETS USING YOHKOH SOFT X-RAY TELESCOPE
}

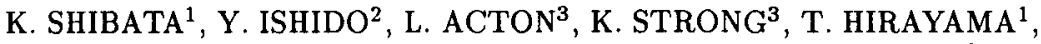 \\ Y. UCHIDA ${ }^{4}$, A. MCALLISTER ${ }^{4}$, R. MATSUMOTO ${ }^{5}$, S. TSUNETA ${ }^{6}$, \\ T. SHIMIZU ${ }^{6}$, H. HARA ${ }^{6}$, T. SAKURAI ${ }^{1}$, K. ICHIMOTO $^{1}$, Y. NISHINO ${ }^{1}$, \\ and Y. OGAWARA ${ }^{7}$ \\ 1 National Astronomical Observatory, Mitaka, Tokyo 181, Japan \\ ${ }^{2}$ STE Laboratory, Nagoya University, Toyokawa 442 \\ ${ }^{3}$ Lockheed Palo Alto Research Laboratory, Palo Alto, U.S.A. \\ ${ }^{4}$ Department of Astronomy, The University of Tokyo, Bunkyo-ku, Tokyo \\ ${ }^{5}$ College of Arts and Sciences, Chiba University, Chiba 260 \\ ${ }^{6}$ Institute for Astronomy, The University of Tokyo, Mitaka, Tokyo 181 \\ 7 Institute of Space and Astronomical Science, Sagamihara 229
}

\begin{abstract}
Time series of SXT (Soft X-ray Telescope) images have revealed many jet-like features in the solar corona. Typical size of the "jet" is $5 \times 10^{3}-$ $4 \times 10^{5} \mathrm{~km}$, the typical projected velocity is $30-300 \mathrm{~km} / \mathrm{s}$, and the kinetic energy estimated to be $10^{25}-10^{28} \mathrm{erg}$. Many of the jets are associated with flare-like bright points or sub-flares. Three typical examples are discussed, including an $\mathrm{X}$-ray jet identified with an $\mathrm{H} \alpha$ surge. It is suggested that magnetic reconnection is one of the possible mechanisms to produce these $\mathrm{X}$-ray jets.
\end{abstract}

\section{INTRODUCTION}

The soft X-ray telescope (SXT) aboard Yohkoh has revealed many jet-like features, i.e., collimated features with apparent motion along the direction of collimation. Although it has not yet been established (except for an X-ray jet associated with an $\mathrm{H} \alpha$ surge) that these X-ray "jets" are real jets showing Doppler velocity, it is very likely that they are real jets with mass motion, as will be discussed later.

Here we report the discovery of such X-ray jet-like features (hereinafter simply called jets) using SXT aboard Yohkoh. (More detailed discussion will be given in Shibata et al. [1992b].)

\section{OBSERVATIONS}

We found more than 10 jets per month in the full frame images (FFI) of SXT (Tsuneta et al. 1991) during the period of November 1991 - June 1992. The total number of X-ray jets exceeds 100. The spatial resolution of FFI images is either half resolution $\left(\sim 5^{\prime \prime}\right)$ or quarter resolution $\left(\sim 10^{\prime \prime}\right)$. The time resolution depends on the event, ranging from a few minutes to an hour. We also found several jets in partial frame images (PFI) of 
SXT, whose spatial and temporal resolutions $\left(\sim 2.5^{\prime \prime}\right.$ and $\left.30 \mathrm{sec}\right)$ are much better than those of FFI.

Typical size of the "jet" is $5 \times 10^{3}-4 \times 10^{5} \mathrm{~km}$, the translational velocity is 30 $300 \mathrm{~km} / \mathrm{s}$ (in a few cases, the velocity reached $600-1000 \mathrm{~km} / \mathrm{s}$ ), and the kinetic energy based on the translational velocity is estimated to be $10^{25}-10^{28} \mathrm{erg}$. Many of the X-ray jets are associated with the flaring bright points, or sub-flares.

\section{TYPICAL EXAMPLES OF X-RAY JETS}

1) "Jet" ejected from subflares (flaring active regions): Figure la shows a typical example of a jet ejected from a subflare, which occurred in the active region NOAA 6918 on 1991 Nov. 12 at 11:28 - 11:36. There is no jet in 10:45 data, and the jet disappeared at 13:08. The length and width of the jet are about $2 \times 10^{5} \mathrm{~km}$ and 20000 $\mathrm{km}$, respectively, and the lower limit of the velocity of the jet is $\sim 100 \mathrm{~km} / \mathrm{s}$.

It is interesting to note that a void appears at the footpoint of the jet after its ejection (i.e., during 11:36-13:15). It seems that there is less hot or dense plasma in the void. This void is similar to dark regions often seen in SXT just above sunspots, and seems to be produced by magnetic reconnection occurring during the ejection of the jet.

Is this jet-like feature a real flow of plasma? In order to answer this question, we measured the temperature and the electron density. Using the filter ratio method (Hara et al. 1992), we find the average temperatures of the jet and the ambient corona are nearly the same $\sim 3 \times 10^{6} \mathrm{~K}$. This means that the high X-ray intensity in the jet region is attributed to the increase in density, not due to the increase in temperature. This rejects the possibility that the "jet" is a temperature "wave".

From emission measure analysis, the average electron density in the jet is estimated to be $4 \times 10^{8} \mathrm{~cm}^{-3}$, which is larger than the ambient coronal density by at least a factor 2 . If the increase in the density is due to a wave, it must be the shock * because the density increase ratio $(\sim 2)$ is much larger than 1 . In this case, the plasma flow velocity $\left(v_{\text {flow }}\right)$ just behind the shock is

$$
v_{\text {flow }}=[(a-1) / a] v_{\text {shock }}
$$

where $a=n_{\text {postshock }} / n_{\text {preshock }}=n_{\text {jet }} / n_{\text {cor ona }}$ is the density increase ratio across the shock and

$$
v_{\text {shock }}=(2 a /[(\gamma+1)-(\gamma-1) a])^{1 / 2} C_{s}
$$

is the propagation velocity of the shock front, $C_{s}\left(\sim 200 \mathrm{~km} / \mathrm{s}\right.$ for $\left.T \sim 3 \times 10^{6} \mathrm{~K}\right)$ is the sound speed. For $\gamma=1-5 / 3$, the flow velocity becomes $0.7-0.9 C_{s}$ if the density ratio $a \sim 2$. Hence the density ratio $a \sim 2$ suggests the presence of a real plasma flow whose velocity is of order of the sound speed $(\sim 200 \mathrm{~km} / \mathrm{s})$, even if the density increase is due to the shock.

We can also calculate the mass, the kinetic and internal energies of the jet; $M_{\text {jet }} \sim$ $2 \times 10^{13} \mathrm{~g}, E_{k} \sim(1 / 2) M_{\text {jet }} v^{2} \sim 10^{27} \mathrm{erg}$, and $E_{\text {int }} \sim 10^{28} \mathrm{erg}$. Since the total mass of

* From the global magnetic field configuration around the jet, we assume that the plasma flow is along the magnetic field so that the shock (= slow mode MHD shock) can be treated as a pure hydrodynamic shock. The essential point of the discussion (especially the conclusion), however, is quite general even for more general slow/fast MHD shocks, in which case we have to use more complex MHD shock formulae. 
the jet is comparable to the initial mass contained in the footpoint active region and the active region mass increased during the ejection of the jet, the mass must be supplied from somewhere, probably from chromosphere via evaporation (Hirayama 1974) during the subflare at the footpoint of the jet. The observed velocity and density distribution of the jet supports a model of the evaporation flow occurring in an open flux tube (or in a very long loop); the energy of the evaporation flow seem to be provided by magnetic reconnection between emerging flux and the vertical coronal field.

2) "Jet" with undulating or meandering configuration: Figure 1b shows a typical example of the jet with meandering or undulating structure occurred on 1991, Nov. 8 . The length of the jet is $\sim 4 \times 10^{5} \mathrm{~km}$ at $04: 10$, and the lower limit of the translational velocity of the jet is $\sim 110 \mathrm{~km} / \mathrm{s}$.

It is very impressive that the jet shows the meandering or undulating configuration, which suggests that the jet may be along the helically twisted magnetic field lines. One of the possible mechanisms of this class of jet is the "magnetic-twist" mechanism (Shibata and Uchida 1986), where the magnetic twist is suddenly released by magnetic reconnection from twisted flux tube to untwisted tube, and such twist accelerate plasmas by $\mathbf{J} \times \mathbf{B}$ force.

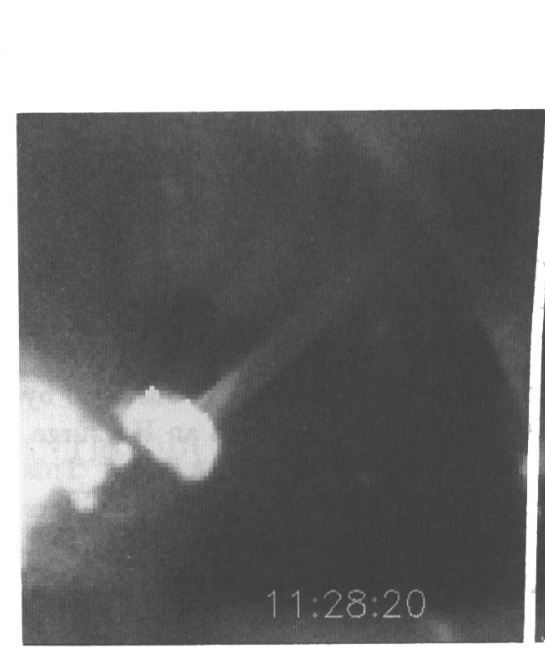

(a)

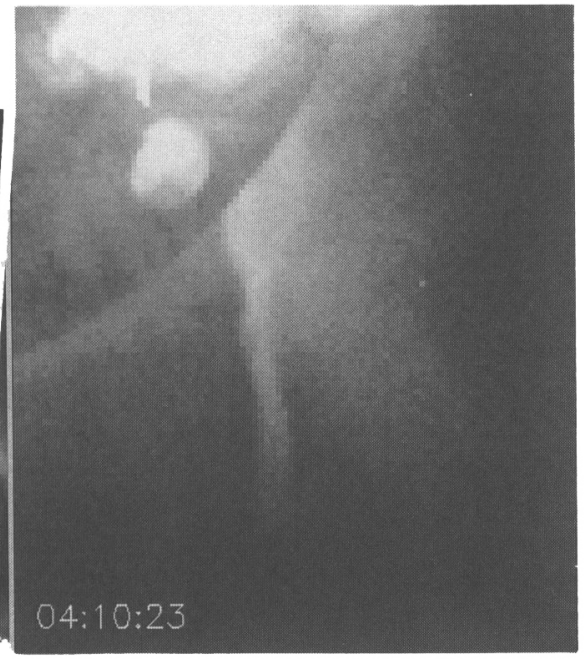

(b)

Fig. 1. (a) Typical example of the $\mathrm{X}$-ray jet associated with a subflare, which was observed at 11:28 UT on Nov. 12,1991 , in active region NOAA 6918 . The size of each image is $120 \times 120$ half resolution pixels, i.e., $600^{\prime \prime} \times 600^{\prime \prime}$. (b) An X-ray jet with meandering or undulating configuration, which was observed at 04:10:23 UT on 8-Nov-91. This image suggests the presence of helical magnetic field along the jet. The size of the image is $950^{\prime \prime} \times 1100^{\prime \prime}$.

3) "Jet" identified with an $\mathrm{H} \alpha$ surge: Figure 2 shows the $\mathrm{X}$-ray image of the jet identified with an $\mathrm{H} \alpha$ surge, as well as the $\mathrm{H} \alpha$ image of the surge taken by the Flare Telescope at Mitaka (Sakurai et al. 1992). The comparison of these images has revealed 
that the X-ray brightening occurred at the footpoint of the surge, where $\mathrm{H} \alpha$ brightening also occurred. The direction of elongation of the $\mathrm{X}$-ray jet is approximately the same as that of the surge. There is no strong $X$-ray emission at the top of the surge. The mass and the kinetic energy of the X-ray jet are estimated to be $\sim 2 \times 10^{11} \mathrm{~g}$ and $10^{25} \mathrm{erg}$, respectively, which are much smaller than those $\left(10^{14} \mathrm{~g}\right.$ and $\left.10^{28} \mathrm{erg}\right)$ of the $\mathrm{H} \alpha$ surge.

The discovery of coexistence of the $\mathrm{X}$-ray emission and $\mathrm{H} \alpha$ emission in a surge gives a strong constraint on the theory of surges. The simple picture of pure hydrodynamic acceleration along rigid magnetic flux tube seems to be no longer valid for this class of surge. This is because if we input a large amount of energy to the upper chromosphere to make an X-ray emitting plasma, then the entire cold plasma above the energy injection point are heated up and evolve into X-ray emitting plasma (i.e., evaporate), so that there is no cold plasma jet emitting $\mathrm{H} \alpha$ (Sterling et al. 1993). In order to make both hot and cold plasmas, we have to assume multi-dimensional magnetic field structure. Shibata et al. (1992a, 1993) showed that the mixture of hot and cold plasmas are ejected along the current sheet as a result of magnetic reconnection between emerging flux and pre-existing coronal fields.

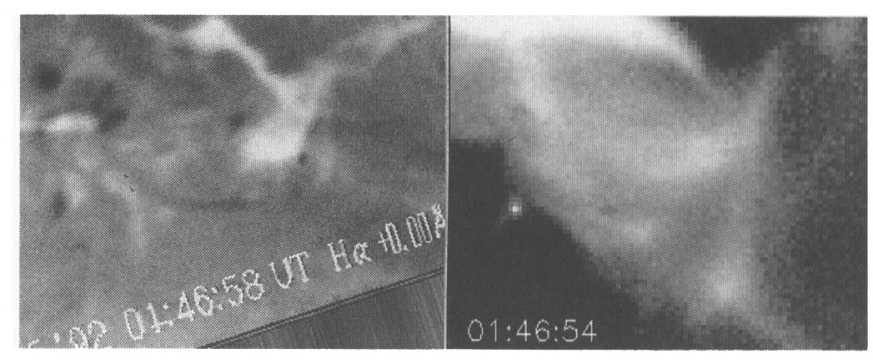

Fig. 2. Left: $\mathrm{H} \alpha$ image of the surge of Feb. 25, 1992, 01:46:58 UT, observed by the Flare Telescope at Mitaka. Right: An X-ray jet identified with an $\mathrm{H} \alpha$ surge, which occurred on Feb. 25, 1992, 01:46:54, near the flare in NOAA 7070. This image is taken with partial frame image mode at full resolution $\left(\sim 2.5^{\prime \prime}\right)$; filters and exposure times are $\mathrm{Al} / \mathrm{Mg} / \mathrm{Mn}$ and $17.0 \mathrm{msec}$. The image size is $95 \times 77$ pixels, i.e., $238^{\prime \prime} \times 193^{\prime \prime}$. The $\mathrm{H} \alpha$ image is approximately coaligned with the SXT image.

\section{REFERENCES}

Hara, H. et al.: 1992, Publ. Astron. Soc. Japan, 44, in press

Hirayama, T.: 1974, Solar Phys., 34, 323

Sakurai, T. et al:: 1992, Publ. Astron. Soc. Japan, 44, L7

Shibata, K. and Uchida, Y.: 1986, Solar Phys., 103, 299

Shibata, K., Nozawa, S., and Matsumoto, R.: 1992a, Publ. Astron. Soc. Japan, 44, 265

Shibata, K., Nozawa, S., and Matsumoto, R.: 1993, in these proceedings

Shibata, K. et al.: 1992b, Publ. Astron. Soc. Japan, 44, in press.

Sterling, A. C., Shibata, K., and Mariska, J. T.: 1993, Astrophys. J., in press.

Tsuneta, S. et al.: 1991, Solar Phys., 136, 37. 